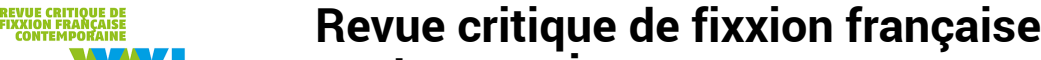 \\ XXI contemporaine

\section{L'invisibilité et la marginalité comme formes de résistance paradoxale au pouvoir}

Alikavazovic, Damasio, Despentes, Rosenthal, Vasset

Frédéric Martin-Achard

\section{OpenEdition}

\section{Journals}

Édition électronique

URL : https://journals.openedition.org/fixxion/459

DOI : $10.4000 /$ fixxion.459

ISSN : 2295-9106

\section{Éditeur}

Ghent University

Référence électronique

Frédéric Martin-Achard, «L'invisibilité et la marginalité comme formes de résistance paradoxale au pouvoir 》, Revue critique de fixxion française contemporaine [En ligne], 21 | 2020, mis en ligne le 15 décembre 2020, consulté le 17 février 2022. URL : http://journals.openedition.org/fixxion/459 ; DOI : https://doi.org/10.4000/fixxion.459

Ce document a été généré automatiquement le 17 février 2022.

\section{(c) (i) (3)}

Les contenus de la Revue critique de fixxion française contemporaine sont mis à disposition selon les termes de la licence Creative Commons Attribution - Pas d'Utilisation Commerciale - Pas de Modification 4.0 International. 


\title{
L'invisibilité et la marginalité comme formes de résistance paradoxale au pouvoir
}

\author{
Alikavazovic, Damasio, Despentes, Rosenthal, Vasset
}

Frédéric Martin-Achard

"Disparaissons"! Cette injonction à l'évanouissement constitue le titre du deuxième ouvrage du bien nommé Comité invisible. En regard de la première page du chapitre se trouve une photographie d'une inscription murale au pochoir répétant le même mot d'ordre, mais en allemand Lass uns verschwinden, et légendé "Istanbul, juin 2013"1. La référence au mouvement protestataire turc initié cette année-là autour du parc Gezi dans le quartier de Taksim fait de l'incitation à disparaître un mot d'ordre politique. L'hypothèse que je souhaite soumettre ici est que ce mot d'ordre est celui de tout un pan de la fiction romanesque française de ces mêmes années, qui met en scène des formes de résistance au pouvoir, lesquelles se caractérisent avant tout par la marginalité et l'invisibilité. La marginalité et l'invisibilité n'y sont pas subies, mais intentionnelles, et se trouvent au fondement de tout un répertoire d'actions politiques dissidentes. Dans le prolongement des travaux de Dominique Rabaté ${ }^{2}$, qui étudie le désir de disparaître qui habite de nombreux personnages du roman français contemporain, je formule donc l'hypothèse que ce motif de la disparition connait, aux environs de 2013, une inflexion collective et plus explicitement politique. La disparition apparait dès lors comme une modalité de résistance et invite à réfléchir à la nature du pouvoir auquel on refuse de se soumettre en s'évanouissant, en organisant son invisibilité.

Dans Désirs de disparaittre, Dominique Rabaté montre l'importance du motif de la disparition dans le roman français de ces dernières décennies. Il en pointe également la fondamentale ambivalence, entre la disparition subie, héritage des pires tragédies de l'Histoire du $\mathrm{xx}^{\mathrm{e}}$ siècle écoulé - dont le roman de Perec constitue en quelque sorte la figure tutélaire - et la disparition volontaire, le souhait d'être introuvable, de vivre hors d'atteinte et sans laisser de traces, qu'on retrouve mutatis mutandis chez Christian 
Garcin, Emmanuel Carrère ou Éric Faye. Cet effacement intentionnel de soi, ajoute Rabaté, ferait du roman "un lieu paradoxal de résistance face à la normalisation sociale, aux dispositifs toujours grandissants de contrôle et d'assignation, une façon de déserter qui puisse exprimer la force encore vitale d'une sécession individuelle"3. Les textes que j'examinerai ici appartiennent davantage à ce second pôle ${ }^{4}$, à la différence que le désir de disparaître n'y est pas une affaire individuelle, mais un projet collectif. Cet infléchissement de l'effacement individuel vers le retrait collectif n'est pas sans conséquence, on le verra, sur le type d'injonctions et de pouvoir auquel on tente de se soustraire. S'il faut le dater, je le situerais autour de 2013, avec la publication de La conjuration de Philippe Vasset comme terminus a quo. Dans le sillon du roman de Vasset, j'étudierai donc le développement de ces formes de résistance fictionnelle caractérisée par la marginalité et l'anonymat dans L'avancée de la nuit de Jakuta Alikavazovic, la trilogie Vernon Subutex de Virginie Despentes, Éloge des bâtards d'olivia Rosenthal et Les furtifs d'Alain Damasio.

\section{La conjuration : les échecs de la disparition individuelle}

Dans La conjuration, le passage d'une sécession individuelle à une dissidence collective est marqué par l'articulation entre les deux parties du texte. En effet, la première partie est entièrement narrée à la première personne. De retour à Paris après trois ans d'absence, le narrateur constate que les zones autrefois "blanches", friches, immeubles vides et terrains vagues, sur lesquels il vivait "des vies parallèles et rêvées" ${ }^{\text {, s sont }}$ désormais comblés, bétonnés, construits et réaffectés. Or, ces lieux constituaient pour le narrateur des échappatoires, des possibilités de fuite, des doubles fonds dans le réel autorisant "une existence en suspens", permettant "de considérer qu'elle n'était, après tout, qu'un choix parmi d'autres, que rien n'était définitif et la fuite toujours possible" (C 14). Cette "existence en suspens" étant désormais impossible, le narrateur se trouve sommé de rentrer dans le rang, d'adopter une forme de vie unique et standardisée :

Je me retrouvais pris dans la nasse d'une vie étrangère, une vie où il fallait voter, travailler et aimer, une vie où l'on est tenu de s'engager, de prendre ses responsabilités et d'avoir des opinions, une vie régie par les règles de la propriété et $\mathrm{du}$ respect de la vie privée, une vie d'horaires, de rendez-vous et de tâches à effectuer. Fini de jouer : c'était ici et maintenant que les choses se passaient. J'étais prié de faire des choix. (C 15)

Ces injonctions font naître chez le narrateur un désir de se soustraire "à la pesanteur d'être soi" (C 54), qui se manifestera par diverses activités toutes caractérisées par des formes d'invisibilité et d'abandon de soi : être prestataire pour un romancier, pour lequel il enquêtera en vue de fonder une secte, pratiquer la filature, s'introduire anonymement dans des événements privés. Il se tourne également du côté de l'écriture, mais en vain :

Écrire avait, pour moi, quelque chose à voir avec l'invisibilité : c'était disparaître pour n'être plus qu'une parole qui suinte des murs, un bourdonnement mêlé aux bruits de la ville, un goût de fumée affleurant soudain sur les lèvres. Écrire, c'était les nerfs sans le corps, le trajet sans l'identité, la feinte, le vol et l'effraction. C'était échapper, toujours, sans cesser d'être là, jamais. (C 21)

5 Si l'entreprise scripturaire échoue, faute d'un nécessaire effacement de soi, elle pointe avec des accents blanchotiens, le paradigme dominant la première partie du roman : l'écriture, comme les formes de vie successivement adoptées, sont autant d'exercices de 
déprise identitaire, de résistance aux assignations à être soi. En 1998 déjà, Alain Ehrenberg montrait, dans La fatigue d'être soi, que l'apparition de nouvelles normes prônant la responsabilité et la liberté individuelles, l'autonomie et le choix avaient entraîné des mutations de l'individualité contemporaine, faisant de la réussite personnelle une nécessité et de la réalisation de soi un impératif. Pour le narrateur, il s'agit bien de résister à "l'injonction à devenir soi-même" pointée par Ehrenberg', c'està-dire que la première partie du roman se situe encore dans le paradigme individuel qui fait de la disparition une quête personnelle parfois teintée de métaphysique. La bascule est annoncée dès la première partie: la ville que le narrateur retrouve après son absence, devenue une incarnation de la société de contrôle, hérissée de caméras et de signaux GPS, fait naître en lui un projet de "subversion urbaine" ( $C 53)$.

\section{La bascule du singulier au collectif : vers un nouveau paradigme}

6 Or, ce projet prend forme dans la seconde partie du livre, constituée de brefs chapitres, lesquels sont précédés d'un paragraphe en italique énonçant les règles d'un mouvement, la Conjuration, sur le modèle d'une règle monastique ${ }^{7}$. Cette seconde partie marque simultanément la sortie du romanesque et une rupture énonciative : les temps du récit sont remplacés par ceux du discours (présent et futur) et le "je" de la première partie alterne avec d'autres instances pronominales (le "on" de la communauté indéterminée, le "ils" des conjurés ou un "vous" prescriptif), jusqu'à ce que son éviction soit thématisée 8 . Au fil des chapitres se constitue donc la Conjuration, groupe subversif dont le but unique, comme l'énonce la règle numéro deux, est la disparition. Le conjuré abandonne tous les attributs de son identité personnelle, se défait de tous les oripeaux de sa vie sociale et se voue à "disparaitre au monde et à luimême" (C 203). Mais la disparition prônée par la Conjuration se fait au vu et au su de tous, elle "implique de devenir si peu remarquable que personne ne pourra jamais se souvenir de vous avoir vu" ( $C 181)$. Aussi les conjurés arpentent-ils la ville dans le plus complet anonymat, occupant les bureaux vides la nuit, se fondant dans la foule, se glissant dans les interstices de l'espace urbain. Nulle volonté de fuir donc, plutôt d'occuper le terrain tout en échappant aux dispositifs de surveillance et aux assignations à la visibilité et à la traçabilité.

Entre les deux parties du roman a donc lieu la bascule d'un paradigme individuel à une forme d'organisation collective, mais aussi d'une injonction à l'épanouissement personnel à un régime de surveillance généralisée. On la trouve aussi, de façon presque concomitante, entre les deux premiers essais du Comité invisible. En effet, si L'insurrection qui vient raille les exhortations publicitaires à être soi-même et "[1]'injonction, partout, à 'être quelqu'un"', leur opposant un Moi en grève, "un pas de côté vers une désaffiliation politique"10, À nos amis fait d'une part le constat d'une surveillance diffuse et omniprésente - par l'exploitation des "données personnelles" que produit en permanence l'individu hyperconnecté - et d'autre part appelle donc à une disparition collective et au rétablissement d'une forme de conflictualité ${ }^{11}$ au sein de l'individu comme de la société. Or, c'est ce nouveau paradigme du pouvoir diffus et de l'omnisurveillance qui va essaimer dans plusieurs romans à partir de La conjuration. 


\section{Technologie de surveillance et anonymat (Alikavazovic, Despentes)}

Dans L'avancée de la nuit de Jakuta Alikavazovic, la question du pouvoir, de la peur et de la surveillance qu'elle justifie constitue une toile de fond, omniprésente même si parfois de façon diffuse, à l'histoire d'amour entre Paul et Amélia, puis à la paternité de celui-ci. L'histoire entre les deux protagonistes commence par l'entremise d'écrans de surveillance, puisqu'Amélia loge dans l'hôtel Elysse ${ }^{12}$ qui emploie Paul comme veilleur aux heures creuses. La relation amoureuse se déploie ensuite, jusqu'à son épuisement ou plutôt jusqu'à la fuite d'Amélia, dans une atmosphère proche du roman d'anticipation, saturée de paranoïa et de contrôle. Le roman multiplie les dispositifs de surveillance: Paul fait fortune dans la sécurité, Amélia capte des images de zones de guerre au moyen d'un drone ${ }^{13}$, tandis que leur mentor à tous deux, la professeure d'université Anton Albers, brosse dans ses cours l'image de villes tiraillées entre fantasmes sécuritaires et peurs paniques. Mais c'est surtout Louise, la fille de Paul, qui se trouve confrontée à tous les dispositifs de contrôle dans un Paris tétanisé, hanté par la menace terroriste :

Louise et son ami David grandirent dans une ville terrifiée, paralysée par son propre reflet, morcelée sur une multitude d'écrans de surveillance, dont aucun pourtant ne sut prévenir les attaques foudroyantes, un camion lancé dans la foule, un homme flambé à l'essence dans une salle de cinéma (AN 222).

Les jeunes gens font face à un pouvoir répressif et violent, mais peu défini dans ses structures et dont les manifestations principales dans le roman sont la surveillance et la répression armée. Louise et son compagnon, surnommé Le Délavé, développent divers modes de résistance fondés sur l'anonymat, le camouflage et l'invisibilité, qui ne sont pas sans rappeler les stratégies des conjurés. Mais l'une des forces du roman est sans doute de montrer comment, par le biais de la peur, le pouvoir répressif se diffuse et se trouve intériorisé par tout un chacun. Ainsi, Paul fait implanter une puce GPS dans le bras de sa fille, à son insu, pour suivre en temps réel ses déplacements. Lui qui écoute, mi-admiratif, mi-narquois, les exploits de sa fille en matière d'invisibilité, de déguisement et de dissimulation, et qui rêve au début du roman à une fusion avec son environnement, un camouflage dans lequel il entrevoit un bonheur possible ${ }^{14}$, se fait pourtant l'agent d'un pouvoir oppressif, mais horizontal, collaboratif et diffus. Là encore, c'est la peur comme sentiment parental dominant qui fait de Paul un relai efficace et consentant de la surveillance et du traçage, comme le théorise Anders, pour qui l'amour pour les enfants constitue "un cheval de Troie" :

L'amour pour nos enfants est la façon dont un monde indéfendable paraît défendable et est, pour finir, défendu. Accueilli. Les mensonges. La surveillance globale. La militarisation insidieuse. Qui ne voudrait pas savoir ses enfants en sécurité ? Qui n'accepterait pas de payer le prix fort pour cela ? C'est par amour que nous équipons nos villes, nos rues et nos maisons. (AN 202)

10 Ainsi, par amour pour sa fille, Paul lui implante une balise GPS et suit ses pérégrinations nocturnes sur un écran. Il faut dire qu'un étrange phénomène se répète en deux occurrences et à deux époques distinctes dans le roman, dans le récit que livre Amélia de son enfance d'abord puis dans l'enfance de Louise : "les filles disparaissaient, il sévissait une hémorragie, non, une évaporation d'adolescentes, un soir elles se couchaient dans leur lit et le lendemain n'y étaient plus"15. Toutefois, malgré les précautions de son père, sa fille n'échappe pas à cet évanouissement collectif : elle se 
débarrasse de la puce GPS, dont elle avait connaissance, et s'échappe. Pour Louise, qui n'avait jamais été "dans une pièce sans écran" (AN 236), comme pour tous les "jeunes gens tristes"16 de sa génération, la dernière résistance possible repose sur une forme de vie proche de celle des conjurés de Vasset : "échapper à ce regard permanent qui pesait sur tout, tout le temps" (AN 242) en occupant les lieux vides la nuit, l'envers de la ville, en évoluant "dans les interstices, les ombres, les failles" (AN 223).

11 Sécession collective et recherche d'anonymat sont des motifs qui parcourent également la trilogie Vernon Subutex de Virginie Despentes. Dans les trois romans, comme chez Vasset ou Alikavazovic, se crée une communauté en rupture, dans les marges de la société. S'agrègent en effet, progressivement et spontanément, autour du héros éponyme, centre vide de la trilogie, des individus qui finissent par vivre dans un camp itinérant avec pour seul but la tenue d'événements festifs nommés les "convergences". Pour assister aux convergences, il faut au préalable s'être rendu introuvable, "intraçable" en abandonnant tous les objets connectés susceptibles d'émettre des signes repérables de sa présence. Pour les membres permanents de la communauté, "il s'agit de disparaître. Sécurité sociale compte bancaire identité digitale abonnements impositions assurances cartes grises" ${ }^{17}$. Les personnages de la trilogie prennent peu à peu conscience de l'importance de se défaire ou à tout le moins de contrôler cette "vie numérique" qui redouble en permanence leur vie. La communauté se forme d'abord autour de l'usage des "réseaux sociaux" avant de chercher à s'en défaire. Contrairement à L'avancée de la nuit, la trilogie Vernon Subutex nomme le pouvoir dont il s'agit de se défaire : celui des programmes de surveillance tels que PRISM mis en place par la NSA, celui des réseaux sociaux qui conservent des bases de données considérables sur leurs usagers, mais aussi celui des classes dominantes incarnées par le producteur Laurent Dopalet et sa violence notamment à l'encontre des femmes. La trilogie de Despentes est donc en apparence plus explicitement politique que les deux exemples précédents: les monologues narrativisés ou intérieurs ${ }^{18}$ des différents personnages, qui constituent l'essentiel des romans, balaient tout le spectre des opinions politiques, de l'extrême gauche à l'extrême droite. Pour autant, le montage ou la confrontation de tous ces points de vue souvent violemment antagonistes se résout dans un utopique "décrochage collectif" (VS III 312). La trilogie identifie les maux qui gangrènent le monde occidental néo-libéral, mais leur oppose une communauté éphémère fondée sur la danse, la douceur (VS III 126-127) et l'anonymat. La conflictualité apparente des points de vue s'annihile dans la transe provoquée par la musique et l'empathie avec laquelle Despentes traite chacun de ses personnages: les convergences représentent plus une esquive qu'une résistance au pouvoir, une échappatoire festive et évanescente dans des "interstices" (VS III 143) du monde social.

\section{Résistance et communauté empathique : Éloge des bâtards d'Olivia Rosenthal}

Les neuf personnages qui peuplent Éloge des bâtards d'Olivia Rosenthal se constituent en groupe, en microcommunauté qui finit par choisir, sous l'impulsion maintes fois répétée de la narratrice Lily, de se nommer "les bâtards", parce que "[t]oute la littérature est travaillée par des histoires de bâtards" 19 et qu'ils charrient tous, à différents degrés, des ascendances et des histoires familiales compliquées. Ces personnages évoluent dans un univers fictionnel qui procède de l'anticipation 
dystopique, mais qui ne présente qu'un faible écart le réel. La situation initiale présente des similarités frappantes avec les romans de Vasset ou Alikavazovic: la ville est en passe d'être intégralement bétonnée et policée et ses habitants vivent sous le regard permanent des patrouilles de miliciens, des caméras et des drones :

Et il devait y avoir aussi de petites machines plus discrètes qui volaient entre les immeubles comme de fines libellules. Elles enregistraient des millions d'images privées qui étaient ensuite diffusées et retransmises quelque part dans un bureau étroit, sombre et bas de plafond. La ville était sillonnée d'yeux humains ou mécaniques. On pouvait compter sur l'inflation des vidéos récupérées pour ne pas être repérés comme on comptait jadis sur le nombre de livres dans les bibliothèques pour passer l'air de rien de petits messages fantomatiques entre les pages cornées d'un vieux dictionnaire. (ÉB 120)

Aussi, comme l'explique la narratrice dans le préambule du roman, des individus qui “circulent furtivement la nuit" (ÉB 16), se réunissent peu à peu et mènent la nuit "des actions clandestines destinées à empêcher la disparition complète de [leur] ville" et à "maintenir les derniers espaces en friche, les protéger et en étendre la portée" 20 (ÉB 17). Ces insurgés, qui maintiennent une certaine forme d'anonymat en utilisant des pseudonymes, mènent des actions de subversion urbaine qui relèvent aussi bien $\mathrm{du}$ sabotage que du happening artistique :

Nous renversons des installations de chantier. Nous volons des uniformes. Nous bousculons des officiels. Nous répandons de l'eau sur les routes juste avant les grandes gelées. Nous interceptons des trente-trois tonnes et nous les renversons sur la chaussée. Nous dirigeons des dirigeables. Nous allumons des lampes ultrapuissantes là où la nuit devrait régner. Nous coupons des alimentations. Nous détournons des circuits. Nous dynamitons les grands ensembles par petites tranches. (Ibid.)

14 Si les pronoms collectifs, le "nous" comme le "on", n'interviennent qu'in fine chez Vasset, prenant progressivement la place du "je" évanescent dans les règles de la Conjuration, le groupe est ici établi dès l'ouverture du roman. De fait, chez Vasset, l'effacement $\mathrm{du}$ "je" et l'épuisement du récit autodiégétique était une condition nécessaire à la subversion collective, tandis que dans Éloge des bâtards c'est le groupe qui rend possible à la fois l'apparition de la voix narrative et l'avènement du récit : Lily, la narratrice, se dit "incapable d'employer [...] la première personne" (ÉB 101) ; seules la constitution d'un groupe et sa nomination lui permettent de "[s]e fondre dans son doux chantonnement" (ibid.). De même, le groupe fait sourdre les récits des différents personnages jalonnant les cinq nuits qui constituent le roman. Or, ces récits de vie prennent rapidement l'ascendant sur la question du pouvoir et des actions séditieuses entreprises par les bâtards. Le roman, comme chez Despentes, mais de façon plus manifeste encore, se détourne de la question politique ou l'aborde de façon oblique, pour se concentrer sur le rapport empathique à l'autre pour fin et moyen ; Lily, douée de télépathie ou de télé-empathie, sans cesse assaillie par la vie des autres, développe une vision consolante de la parole, de l'écoute intime et de la création d'une petite communauté (voir par exemple: ÉB 288 et 309), dont on peut estimer qu'elles constituent des réponses un peu faibles au monde dystopique dans lequel évoluent les personnages.

15 Les actions subversives sont reléguées dans des séquences brèves en italiques, souvent entre les différentes "nuits", après les récits enchâssés. La première séquence est présentée comme un texte intercalaire : ce sont les propositions d'actions des différents personnages, écrites au crayon sur des petits papiers. Ces propositions sont toutes 
énoncées au pronom indéterminé "on" et au conditionnel; la première - "[o]n saturerait la ville de signes invisibles" (ÉB 85 ) - se répétera pour clore les séquences suivantes dans une forme de chiasme. D'une séquence en italique à l'autre s'instaure donc un jeu de répétitions et de variations, parmi lesquelles une variation temporelle. $\mathrm{Au}$ conditionnel succèdent le passé composé ("[o]n a saturé la ville de signes invisibles", $E ́ B$ 136), puis l'imparfait, mais dans une périphrase verbale à valeur d'ultériorité dans le passé (“[o]n allait saturer la ville de signes invisibles”, ÉB 191) et enfin le conditionnel à nouveau (ÉB 263). Parmi ces variations temporelles, le conditionnel et la périphrase verbale à l'imparfait ${ }^{21}$ ont en commun de présenter les actions séditieuses comme appartenant à un futur incertain, vu du passé, le "futur hypothétique" 22 de Guillaume. Malgré le passé composé, accompli du présent, les actes de résistance et de sabotage envisagés s'énonçant initialement et finalement au conditionnel restent nimbés d'un voile d'incertitude, d'une forme d'irréalité. In fine, que ces happenings, instaurant pour la plupart une forme de réenchantement poétique du monde (faire pleuvoir des plumes du haut des tours, défiler en silence avec des porte-voix et des pancartes vierges), aient lieu ou non n'est pas l'essentiel: le conditionnel provoque une forme de décrochage d'avec le réel et c'est cet écart dans l'incertain et le potentiel qui fait figure d'échappatoire. Les signes qui saturent la ville demeurent invisibles; le fait de les imaginer, d'envisager un autre réel possible, constitue en soi un acte de résistance.

\section{Nouvelle forme de vie et mondes possibles : Les furtifs}

Le lien entre un tiroir verbal comme le conditionnel et la résistance à une société dystopique obsédée par la surveillance peut paraître ténu. Toutefois, on le retrouve de façon plus radicale dans Les furtifs d'Alain Damasio. Le roman se déroule au milieu du $\mathrm{xxI}^{\mathrm{e}}$ siècle, dans un monde ultralibéral et sécuritaire ${ }^{23}$ où les principales villes ont été rachetées par des multinationales après leur mise en faillite. Les personnages évoluent dans un "monde bagué où le moindre de leur acte s'enregistre et informe un tiers de ce qu'ils sont et font" ( $F$ 69), "une société de traces, contrôlée jusqu'à l'obscène" ( $F$ 236) comme la qualifie l'une des protagonistes, dans laquelle chaque objet, chaque individu émet de l'information, génère des données. Chez Damasio, grand lecteur de Deleuze, l'influence des réflexions du philosophe sur la "société de contrôle" est patente: la fable est explicitement politique et la nature du pouvoir, tout à la fois autoritaire, liberticide, horizontal et en grande partie privatisé, est plus longuement développée que dans les autres romans qui nous occupent ici.

l'originalité du roman de Damasio est d'avoir imaginé une forme de vie nouvelle, celle des furtifs, ou un concept, la furtivité, qui était en germe dans d'autres romans. Chez Vasset, la conjuration comprend trois niveaux, trois stades d'aboutissements : Discret, Furtif et Absent ( $C 160)$, tandis que les bâtards de Rosenthal ont "une manière bien à [eux], oblique, furtive, de sillonner la ville" (ÉB 22). Dans Les furtifs, il s'agit bien d'une nouvelle forme de vie, ni animale, ni végétale, qui possède "une affinité évidente" avec "un certain rapport à la fuite, à la liberté, au refus du contrôle" (F 308) et dont l'“éthos fondamental [...] est de savoir se cacher" (F 314). D'abord traqués par une unité spéciale au sein de l'armée, les furtifs deviennent rapidement l'objet de toutes les attentions et de tous les fantasmes: pour les résistants au contrôle exercé par les multinationales, ils incarnent "la fuite, l'invisibilité, l'intraçabilité, le brouillage, le flou" (F 387), c'est-à-dire une échappatoire possible au pouvoir, tandis que pour le 
pouvoir répressif, ils constituent une menace à éradiquer et un bouc émissaire idéal pour attiser la peur. Mais plus qu'une forme de vie, le furtif est une force de vie: en constante métamorphose, il ne se fige jamais dans une individualité stable, mais "procède, par sauts, à des individuations ultra-rapides, qui ne lui offrent jamais que des formes transitoires" (F 396), comme l'explique le philosophe Varech citant Simondon. Son existence est avant tout sonore, musicale. Ainsi donc, le furtif échappe simultanément à la surveillance généralisée, à l'hypercontrôle exercé par les pouvoirs publics et privés et à toute assignation identitaire. À mesure que leur savoir sur les furtifs progresse, les personnages découvrent que l'écriture furtive est elle-même cryptique, la plupart des lettres se cachant derrière certaines lettres-écrans, tandis que leur langage est mouvant: les furtifs sembler manifester un soupçon ou une méfiance toute barthésienne à l'égard du langage "qui incise, qui découpe et qui fragmente le monde" (F 623).

$\mathrm{Au}$ fil du roman, on découvre que cette forme de vie est capable d'hybridation avec l'être humain. C'est d'abord Tishka, la fille des personnages principaux du roman, Sahar et Lorca Varèse, qui disparaît pour devenir furtive, puis ses parents eux-mêmes qui amorcent peu à peu une forme d'hybridation. Or, cette hybridation se manifeste, et c'est l'une des originalités du roman, de façon verbale : le langage de Lorca, puis celui de Sahar, qui exercent tour à tour, au même titre que d'autres personnages principaux du roman, des fonctions narratives, prennent le tour mouvant de celui attribué aux furtifs. La volatilité du langage furtif se transmet donc aux êtres humains à mesure de leur hybridation et se caractérise par plusieurs traits stylistiques ${ }^{24}$ : l'inversion de lettres ou de syllabes au sein d'un mot, proches de la verlanisation, la substitution d'une lettre ou d'une syllabe à une autre, ou l'ajout de lettres ou de syllabes au sein d'un mot. La furtivité du langage concerne donc avant tout la morphologie du lexique, la syntaxe demeurant pour sa part intacte ou presque. La labilité des mots provoque aussi bien des recompositions lexicales que des passages plus opaques, proches de la glossolalie. Mais le langage hybride possède une autre caractéristique, grammaticale celle-là : l'abondance du conditionnel. Le "devenir-furtif" de Lorca se dévoile d'abord dans sa faculté croissante, surhumaine, d'échapper à la surveillance ; et cette capacité lui apparaît comme une forme de dédoublement, de clivage, comme si les choses devenaient irréelles. "J'ai juste l'impression de me parler au conditionnel" ( $F$ 281), confie-t-il à Sahar, qui lui fait remarquer que la forme verbale à laquelle il fait référence - en réalité le conditionnel passé - est l'irréel, la forme "qui exprime une hypothèse irréaliste" (ibid.). Le conditionnel, ajoute-t-elle, restitue les trois nuances de la conjugaison latine : irréel du présent, irréel du passé et potentiel. De fait, dans les séquences d'hybridation langagière de Lorca, le conditionnel est le tiroir verbal dominant, et tout particulièrement le conditionnel passé.

Devenir (partiellement) furtif, c'est donc vivre dans une forme d'irréalité, dans un dédoublement que rend possible l'énonciation au conditionnel. En effet, le conditionnel est un temps morphologiquement dédoublé, en ce qu'il cumule des affixes du passé (-ai) et du futur (-r-) pour exprimer l'ultériorité dans le passé; il est aussi fondamentalement dialogique, l'interaction des deux affixes entrainnant un redoublement de l'énonciation principale par une énonciation rapportée ${ }^{25}$. Enfin, comme le futur, il exprime ce qui se réalisera ultérieurement et ouvre dès lors "le champ infini des prolongements possibles" ${ }^{26}$. Mais, alors que le futur restreint "la part d'hypothèse naturellement inhérente à l'avenir, le conditionnel inscrit le procès dans un avenir chargé d'incertitude" ${ }^{27}$. Aussi le conditionnel a-t-il partie liée avec une 
conception ramifiée du temps, c'est-à-dire qui "laisse apparaître l'infinité des 'mondes possibles"' 28 , un monde possible étant "une chaîne de situation dans le temps" 29 . En se vivant et en se racontant au conditionnel, Lorca s'extrait du monde de ce qui est, du monde actuellement réalisé, pour se situer dans un monde possible parmi d'autres. De fait, le début de l'un de ses récits au conditionnel (présent et passé) énonce une suspension temporelle, que l'on peut comprendre comme une sortie du temps linéaire pour ouvrir au champ des possibles: "[i]l y aurait alors comme une suspension du temps" ( $F 450)$. Ce présentatif, loin de correspondre à l'énonciation de ce qui est, comme une sorte de constat thétique, situe la séquence dans un temps ramifié, dans une multiplicité de possibles. À ce titre, il rappelle aussi l'emploi modal du conditionnel "ludique" ou "préludique" au moyen duquel les interactants choisissent une nouvelle identité et qui "qui permet à l'espace transitionnel du jeu de se développer dans le champ des possibles" ${ }^{30}$.

\section{Conclusion : occuper le terrain, investir le temps}

Si ces fictions, qui mettent en scène un pouvoir fondé sur le contrôle horizontal, diffus et permanent de la population, relèvent toutes, à différents degrés, d'une forme d'anticipation ${ }^{31}$ ou de science-fiction, les points de convergence entre elles et avec différents essais par ailleurs, laissent envisager des inquiétudes bien réelles et très contemporaines sur la nature du pouvoir ou des pouvoirs. L'omnisurveillance, le traçage, l'injonction à la transparence mis en scène dans ces fictions ne sont pas sans rappeler le régime de l'“omnivisibilité pathologique" 32 identifié par Pierre Zaoui, la "société d'exposition" ${ }^{33}$ analysée par Bernard Harcourt ou l'"état de surveillance perpétuelle" ${ }^{34}$ que décrit le critique Carlo McCormick en introduction du recueil Surveillance index, edition one. L'intérêt de ces romans est d'exposer ces formes de pouvoir et d'explorer, dans le même temps, les modalités de résistance à ce pouvoir. Elles passent par un répertoire d'actions qui ont toutes en commun la disparition, l'anonymat, l'absence au monde. Toutefois, cette absence ne va pas sans la refondation d'une communauté de marginaux, bâtards, jeunes gens tristes, et autres conjurés, dans une forme d'utopie empathique. Mais surtout, à ce pouvoir omniprésent et omniscient, diffus et intériorisé, voire accepté par chacun, ces romans opposent l'ouverture à d'autres formes de vie, le repli dans des interstices, et l'élargissement du champ des possibles. Si pour les personnages de Vasset, Despentes, Alikavazovic et Rosenthal, la résistance s'incarne avant tout dans la défense et le ré-investissement de l'espace, chez Damasio, à l'occupation du terrain s'ajoute celle d'interstices temporels qui sont autant de mondes possibles dans lesquels échapper au pouvoir. Or, c'est la langue et plus précisément la grammaire qui permet de faire advenir ces mondes possibles, d'ouvrir les personnages à une conception du temps ramifiée. L'énonciation au futur de prophétie ou de prédiction qui clôt $L a$ conjuration envisage déjà l'avenir sous la forme d'un champ des possibles - mais restreint, fermé par la certitude de la victoire future de la conjuration -, tandis que le conditionnel chez Rosenthal et $a$ fortiori chez Damasio laisse ouvertes toutes les conjectures, n'envisage l'avenir qu'en puissance, fait de différents scénarios dont il est loisible pour les personnages de s'emparer. 


\section{NOTES DE FIN}

1. Comité Invisible, À nos amis, Paris, La Fabrique, 2014, p. 132.

2. Voir Dominique Rabaté, Désirs de disparaître. Une traversée du roman contemporain, Rimouski, Tangence, 2015, <Confluences>.

3. Ibid., p. 20.

4. Les textes et les auteurs étudiés dans Désirs de disparaître ne se réduisent pas tous à cette polarité stricte. L'exemple de Villa Amalia de Quignard montre que la disparition d'Ann Hidden, pour intentionnelle qu'elle soit, ne relève pas d'une forme d'héroïsme. De même, les romans de Modiano penchent tantôt du côté de la disparition subie - l'exemple paradigmatique en serait Dora Bruder -, tantôt du côté de la disparition volontaire comme le personnage de Jacqueline/ Loucki du roman intitulé Dans le café de la jeunesse perdue.

5. Philippe Vasset, La conjuration, Paris, Fayard, 2013, p. 11 ; dorénavant $C$.

6. Alain Ehrenberg, La fatigue d'être soi. Dépression et société [1998], Paris, Odile Jacob, 2008, <poches>, p. 14.

7. Une citation de la règle de Saint Benoît figure en exergue de la seconde partie, ne prenant son sens qu'au fil de la lecture.

8. "Il a fallu cesser de dire 'je". (C 192)

9. Comité Invisible, L'insurrection qui vient, Paris, La Fabrique, 2007, p. 14.

10. Ibid., p. 18 (les auteurs soulignent).

11. On remarquera que tandis que le comité invisible en appelle à un individu conflictuel, Michel Biron voit, dans le roman contemporain et chez Michel Houellebecq en particulier, l'essor d'un "personnage non conflictuel", lequel "ne peut jamais que tendre vers son effacement et devenir, malgré lui, une sorte d'abstraction souffrante." (Michel Biron, "L'effacement du personnage contemporain : l'exemple de Michel Houellbecq", Études françaises, vol. 41, n 1, 2005, p. 40). Par un étonnant renversement, l'effacement prôné par le comité invisible devient un acte subversif et participe du rétablissement d'une forme de conflit. C'est à nouveau que le paradigme a changé : la dépression qui affaiblit les structures conflictuelles selon Biron est retournée en Moi en grève, désaffilié volontaire, tandis que l'effacement devient une résistance aux injonctions d'omnivisibilité et aux dispositifs de contrôle contemporains.

12. On notera aussi que le confort de cet hôtel fictif, qui occupe une place prépondérante dans le récit, repose sur "la neutralité, l'anonymat" (Jakuta Alikavazovic, L'avancée de la nuit [2017], Paris, Points, 2018, p. 19 ; dorénavant AN).

13. Dans un essai à charge, Théorie du drone (Paris, La Fabrique, 2013), Grégoire Chamayou fait l'histoire d'une technologie destinée à capter de l'image devenue arme létale, l'histoire "d'un œil devenu arme" (p.22) permettant de "projeter du pouvoir" sans envoyer de troupes dans les zones de guerre, et ses conséquences sur l'éthique de la guerre et la nature du pouvoir.

14. "Il s'était dit qu'ils pourraient se fondre dans les lieux, dans le décor, et que c'était peut-être cela, le bonheur, ou ce qui s'en approchait le plus. Une vaste entreprise de camouflage, avait pensé Paul." (AN 7)

15. AN 80 et 219. Dans la seconde occurrence, la ponctuation est très légèrement modifiée, un point et non un point-virgule après "disparaissaient". Les détails de la disparition changent ensuite d'une occurrence à l'autre, mais le phénomène décrit est le même.

16. Le syntagme revient en plusieurs occurrences dans le roman, par exemple : AN 225 et 232.

17. Virginie Despentes, Vernon Subutex 2 [2015], Paris, Le Livre de Poche, 2016, p. 396 ; dorénavant VS II). Pour les autres volumes : Vernon Subutex 1 [2015], Paris, Le Livre de Poche, 2016 et Vernon Subutex 3 [2017], Paris, Le Livre de Poche, 2018 ; dorénavant respectivement VS I et VS III. 
18. Le monologue intérieur procède à une délégation complète de la narration aux pensées du personnage, tandis qu'avec le monologue narrativisé ces pensées se mêlent à la voix narrative, grâce au style indirect libre. Cette dernière forme énonciative est dominante dans les trois romans de Despentes, mais fait place ponctuellement au monologue intérieur. Sur le monologue narrativisé, voir Dorrit Cohn, La transparence intérieure. Modes de représentation de la vie psychique dans le roman (1978), trad. Alain Bony, Paris, Seuil, 1981, <Poétique>.

19. Olivia Rosenthal, Éloge des bâtards, Paris, Verticales, 2019, p. 197 ; dorénavant ÉB. On aura reconnu l'allusion à Marthe Robert, qui fait de l'arrivée du bâtard l'entrée dans le genre romanesque. Voir Marthe Robert, Roman des origines et origines du roman, Paris, Grasset, 1972.

20. L'analogie avec les ZAD et les zadistes est développée dans le roman, voir ÉB 312-313.

21. L'imparfait du semi-auxiliaire "aller" n'est pas sans évoquer l'imparfait contrefactuel, aussi appelé d'imminence contrecarrée, ce qui tendrait encore plus à déréaliser le procès exprimé.

22. Gustave Guillaume, on le sait, considère le conditionnel non plus comme un mode, mais comme un temps de l'indicatif, le rapprochant du futur mais d'un futur hypothétique. Le conditionnel présente le procès comme très incertain, $d u$ fait qu'il ne s'appuie pas sur une réalité présente, mais sur le passé. Voir Gustave Guillaume, Leçons de linguistique de Gustave Guillaume 1948-1949. Série A. Structure sémiologique et structure psychique de la langue française 1, Québec, Les Presses de l'Université de Laval, Paris, Klincksieck, 1971, p. 135-138.

23. Pour qualifier cette société, l'auteur invente le mot-valise "carcélibérale" (Alain Damasio, Les furtifs, Paris, La Volte, 2019, p. 236 dorénavant $F$ ).

24. Pour un exemple particulièrement frappant, voir le monologue de Lorca : $F 450$.

25. Je me fonde ici sur les travaux de Bres, Azzopardi et Sarasin. Voir Jacques Bres, Sophie Azzopardi et Sophie Sarrazin, "Le conditionnel en français : énonciation, ultériorité dans le passé et valeurs modales", Faits de langues, n 40, 2012, p. 37-43, URL : https://hal.archives-ouvertes.fr/ hal-00780764 (consulté le 11 juillet 2020).

26. Robert Martin et Frédéric Nef, "Temps linguistique et temps logique”, Langages, $\mathrm{n}^{\circ} 64,1981$, p. 12.

27. Robert Martin, "Le futur linguistique : temps linéaire ou temps ramifié ? À propos du futur et du conditionnel français", Langages, $\mathrm{n}^{\circ}$ 64, 1981, p. 85.

28. Robert Martin et Frédéric Nef, art. cit., p. 12.

29. Robert Martin, art. cit., p. 85.

30. Jacques Bres, Sophie Azzopardi \& Sophie Sarrazin, art. cit.

31. Explicite chez Damasio et, dans une moindre mesure, chez Rosenthal, l'anticipation est plus diffuse chez Alikavazovic, le roman mêlant des repères temporels historiques, tels que les guerres des Balkans, et l'évocation d'un futur proche, mais dont l'amplitude reste floue. Chez Vasset, la seconde partie marque un décrochage dans une forme de monde possible, tandis que dans la trilogie de Despentes c'est l'épilogue qui fait basculer le roman dans l'anticipation, envisageant l'avenir des "peuplades des convergences" (VS III 404), évoquant par exemple leurs persécutions dès 2100 .

32. Pierre Zaoui, La discrétion. Ou l'art de disparaître [2013], Paris, Autrement, 2018, <Les grands mots>, p. 144 .

33. Voir Bernard Harcourt, La société d'exposition. Désir et désobéissance à l'ère numérique, Paris, Seuil, 2020, <La couleur des idées>.

34. Le critique décrit un "état de surveillance où la peur a pris le pas sur les préoccupations sociétales et individuelles en matière de vie privée" (" $[\mathrm{A}]$ a perpetual surveillance state where fear has overtaken the societal and individual concerns for privacy", je traduis). Carlo McCormick, "The Eyes Have It", Surveillance Index. Edition One, URL: http://www.surveillanceindex.com/home (consulté le 13 juillet 2020). Le projet Surveillance index réunit deux cents livres de photographies ayant trait aux mécanismes de surveillance. 


\section{RÉSUMÉS}

L'article suit l'hypothèse selon laquelle plusieurs romans parus ces dernières années proposent des formes de résistance au pouvoir, caractérisées avant tout par la marginalité et l'invisibilité. La marginalité et l'invisibilité n'y sont pas subies, mais intentionnelles : elles participent d'un mode d'action et se trouvent même au fondement de tout un répertoire d'actions politiques dissidentes. Cette tendance constitue un infléchissement du " désir de disparaître » étudié par Dominique Rabaté (2015), qui concerne avant tout des personnages individuels: la disparition devient collective et plus explicitement politique dès La Conjuration (2013) de Philippe Vasset, roman qui ouvre la voie à cette inflexion. Si l'invisibilité et l'anonymat apparaissent comme un projet politique subversif, c'est que le pouvoir se manifeste avant tout dans ces romans par le contrôle, la surveillance et une injonction à l'hypervisibilité. La fiction rejoint sur certains points des manifestes politiques comme ceux du Comité invisible, dont l'injonction collective " Disparaissons ! » sert de titre au deuxième chapitre du livre À nos amis (2014). L'étude des formes de résistance au pouvoir dans L'Avancée de la nuit (2017) de Jakuta Alikavazovic, la trilogie Vernon Subutex (2015-2017) de Virginie Despentes, Éloge des bâtards (2019) d'Olivia Rosenthal et Les Furtifs d'Alain Damasio (2019) montre qu'outre l'invisibilité, l'anonymat et l'occupation des interstices du territoire échappant encore à la surveillance, c'est dans la grammaire et le temps que certains romans trouvent l'occasion de faire advenir des mondes possibles pour fuir le pouvoir.

\section{INDEX}

Mots-clés : roman contemporain, disparition, fiction politique, pouvoir, mondes possibles

\section{AUTEURS}

\section{FRÉDÉRIC MARTIN-ACHARD}

Université Jean Monnet de Saint-Étienne 